\title{
Case
}

Report

\section{Complete Resection of a Cavoatrial Metastatic Liposarcoma under Hypothermic Circulatory Arrest}

\author{
Ryumon Matsumoto, ${ }^{1}$ Toshiki Fujiyoshi, ${ }^{1}$ Kentaro Kamiya, ${ }^{1}$ Jun Matsubayashi, ${ }^{2}$ Shoji Fukuda, ${ }^{1}$ \\ Toshiya Nishibe, ${ }^{1}$ and Hitoshi Ogino ${ }^{1}$
}

\begin{abstract}
A patient underwent surgical resection twice for primary and metastatic dedifferentiated liposarcomas. Computed tomography revealed a tumor mass at the cavoatrial junction. Prompt surgical resection of the tumor with thrombectomy was successfully performed using cardiopulmonary bypass with hypothermic circulatory arrest. Despite the poor prognosis of metastatic or recurrent liposarcoma, the patient has survived for 8 years since the first tumor resection.
\end{abstract}

Keywords: dedifferentiated liposarcoma, metastatic tumor, complete resection, cardiopulmonary bypass, hypothermic circulatory arrest

\section{Introduction}

Although liposarcoma is one of the most common soft tissue sarcomas, primary pleural liposarcoma has rarely been reported and its prognosis is reportedly poor due to higher incidences of metastasis and recurrence. ${ }^{1)} \mathrm{We}$ report a case of complete successful resection of a cavoatrial metastatic tumor originating from a primary pleural liposarcoma using cardiopulmonary bypass (CPB) and hypothermic circulatory arrest (HCA). The patient has survived without any metastasis or recurrence.

${ }^{1}$ Department of Cardiovascular Surgery, Tokyo Medical University, Tokyo, Japan

${ }^{2}$ Department of Pathology, Tokyo Medical University, Tokyo, Japan

Received: October 10, 2021; Accepted: December 19, 2021

Corresponding author: Ryumon Matsumoto. Department of Cardiovascular Surgery, Tokyo Medical University, 6-7-1 NishiShinjuku, Shinjuku-ku, Tokyo 160-0023, Japan

Email: ryumon_m@tokyo-med.ac.jp

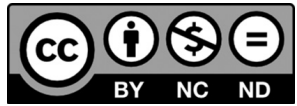

This work is licensed under a Creative Commons Attribution-NonCommercialNoDerivatives International License.

(C)2022 The Editorial Committee of Annals of Thoracic and Cardiovascular Surgery

\section{Case Presentation}

A 68-year-old man was referred to our department for surgical treatment of a tumor at the cavoatrial junction between the inferior vena cava (IVC) and the right atrium (RA). Initially, 6 years prior (at the age of 62), he had undergone left lower lobectomy for lung cancer. However, the final diagnosis of the resected tumor was pleural dedifferentiated liposarcoma because a pathological analysis was positive for the Mouse double minute 2 homolog (MDM2), adipophilin, and perilipin. In one year (at the age of 63), surgical adrenalectomy was performed for the right adrenal tumor, and repeated one year (at the age of 64) later for the left-sided tumor. The pathological examinations of the bilateral adrenal tumors also demonstrated dedifferentiated liposarcoma metastasizing from the primary pleural liposarcoma. Follow-up computed tomography $(\mathrm{CT})$ scans after surgery revealed a mass at the cavoatrial junction, which was initially suspected to be a thrombus in the IVC. Rivaroxaban was administered for deep vein thrombosis. Repeated CT scans revealed enlargement of the mass, which was suspected to be a metastatic liposarcoma (Fig. 1).

The patient was asymptomatic. The white blood cell count and C-reactive protein levels had increased to $12800 / \mu \mathrm{L}$ and $2.0 \mathrm{mg} / \mathrm{dL}$, respectively, and the D-dimer 
Matsumoto $R$, et al.

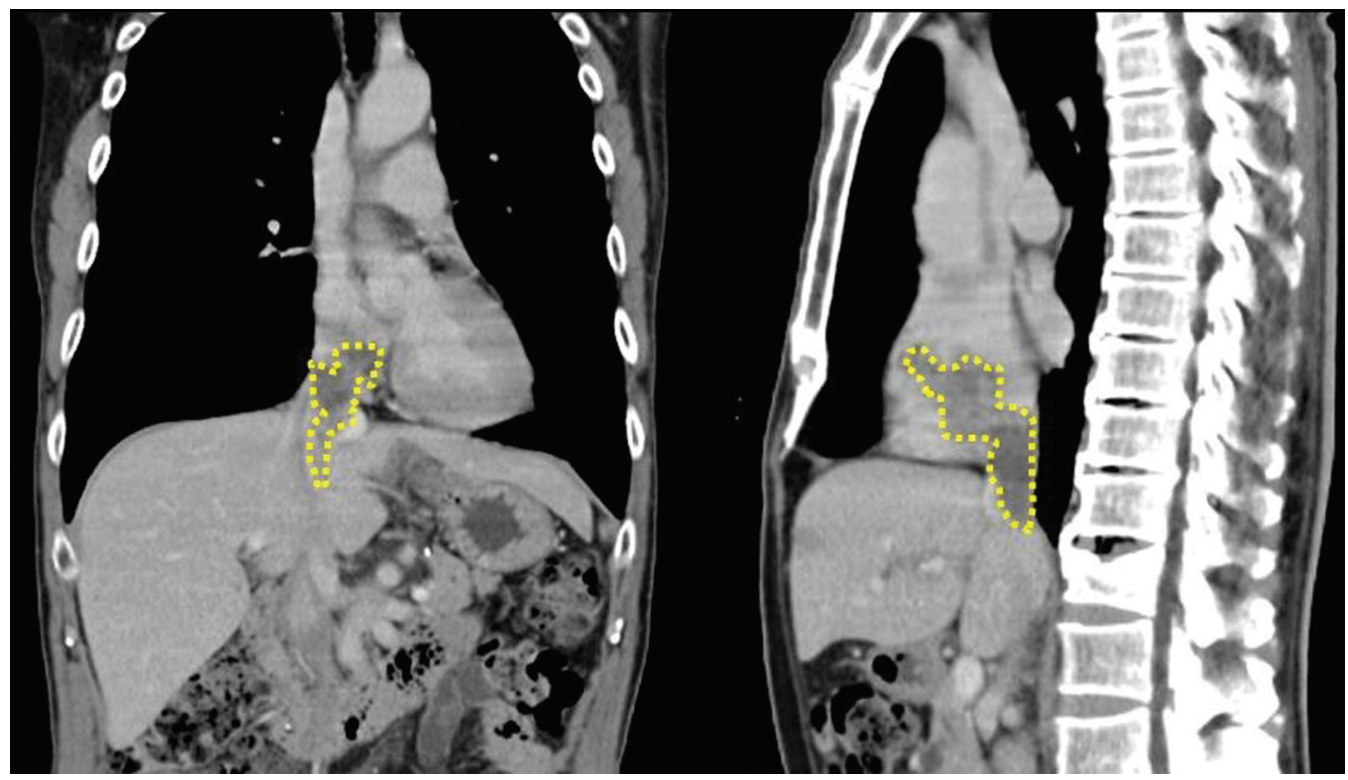

Fig. 1 Preoperative contrast-enhanced CT demonstrated an IVC tumor, which appears to be extending into the RA. CT: computed tomography; IVC: inferior vena cava; RA: right atrium

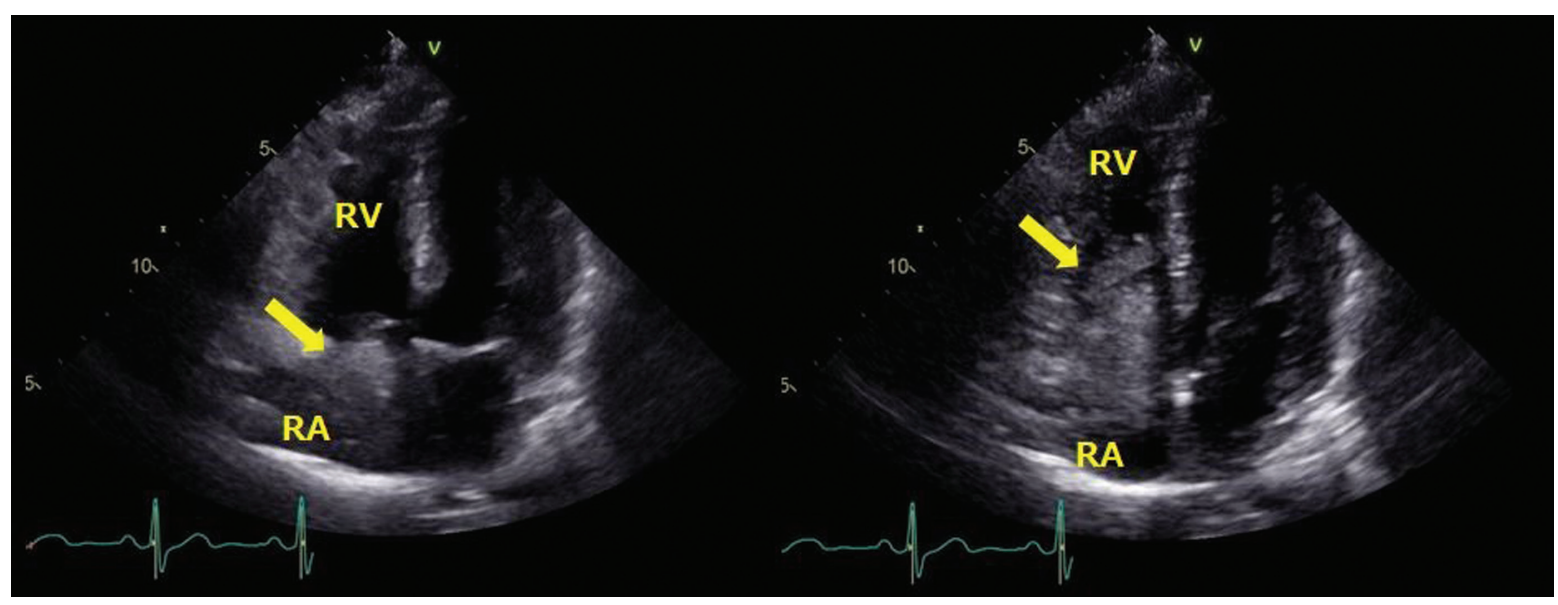

Fig. 2 Preoperative echocardiogram identified a large tumor in the RA that was prolapsing through the tricuspid valve into the RV. RA: right atrium; RV: right ventricle

level was $1.08 \mu \mathrm{g} / \mathrm{mL}$. Tumor markers including squamous cell carcinoma antigen, sialyl-Lewis X, cytokeratin 19 fragment, and pro-gastrin-releasing peptide were negative. Transthoracic echocardiography showed a highly mobile large mass of $60 \mathrm{~mm}$ in diameter in the RA, which extended into the right ventricle through the tricuspid valve (Fig. 2). Coronary angiography revealed chronic total occlusion of the right coronary artery (RCA). Paroxysmal atrial fibrillation was recorded repeatedly during the hospitalization.

\section{Surgery}

On day 8 of the hospitalization, resection of the cavoatrial tumor with thrombectomy, pulmonary vein isolation, and coronary artery bypass grafting (CABG) with a saphenous vein graft (SVG) to the RCA was performed. CPB was established with ascending aortic cannulation and venous drainage from the right femoral vein with a 21-Fr-long cannula and the superior vena cava (SVC). The tip of the femoral vein cannula was carefully adjusted in the proximal part of the IVC under transesophageal echocardiographic guidance to avoid contact with the tumor or thrombus. After successful pulmonary vein isolation for paroxysmal atrial fibrillation, cardiac arrest was induced by antegrade cardioplegia with the ascending aorta clamped. The SVC and IVC were snugged and a right atriotomy was made. A yellowish granular tumor mass was identified in the RA. Due to its distal extension into the IVC beyond the hepatic vein 


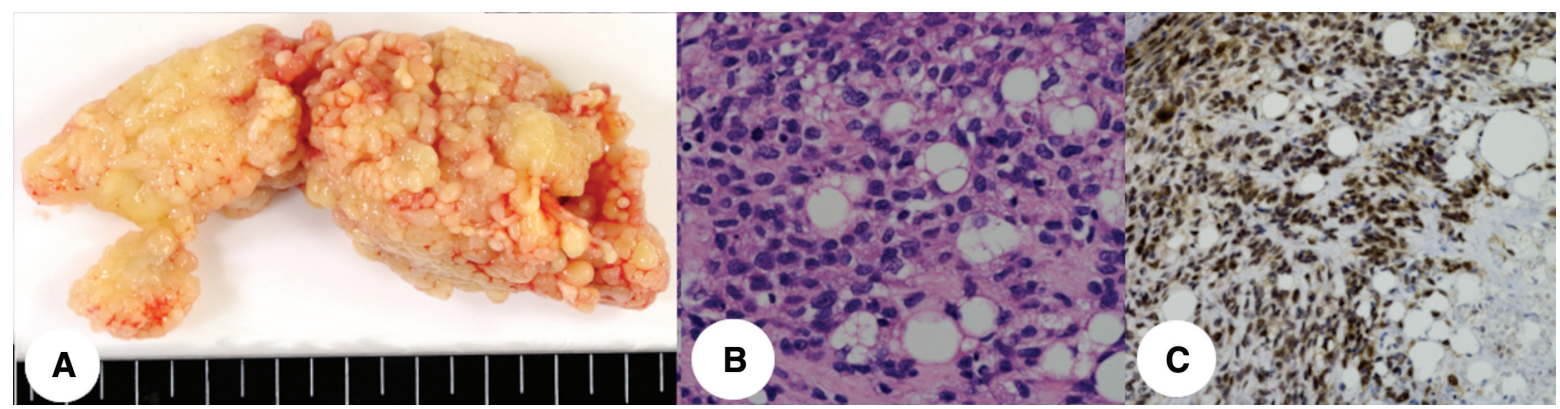

Fig. 3 Macroscopic and microscopic appearance of the extirpated tumor. (A) Macroscopic appearance of the extirpated tumor. (B) Microscopic examination of the lesion demonstrated tumor cells containing lipoblastic cells with vacuolated cytoplasm characteristic of liposarcoma (HE: high-power field). (C) Immunohistochemically, the tumor cells demonstrated diffuse positivity for MDM2 (HE: high-power field). HE: hematoxylin and eosin; MDM2: the Mouse double minute 2 homolog

junction, it was difficult to remove the tumor completely in the bloody surgical field. Hence, core cooling was performed to create a bloodless field under HCA in addition to surface head cooling with ice bags. At the targeted tympanic temperature of $25^{\circ} \mathrm{C}$, the tumor was removed smoothly under HCA. The completeness of the tumor resection was verified endoscopically. The duration of HCA was fortunately only 3 minutes. During rewarming, $\mathrm{CABG}$ to the RCA was performed using an SVG. The patient was smoothly weaned from CPB using a low dose of catecholamine. The HCA, aortic crossclamp, CPB, and operative times were 3, 84, 172, and 304 minutes, respectively.

The resected tumor was composed of pleomorphic tumor cells containing lipoblastic cells with vacuolated cytoplasm. Immunohistochemically, the tumor cells demonstrated diffuse positivity for MDM2 (Fig. 3). The histopathological diagnosis of the tumor from the IVC and RA was dedifferentiated liposarcoma. On postoperative day 14, the patient was discharged.

After discharge, the patient has undergone no postoperative adjuvant chemotherapy or radiotherapy. More importantly, he has been well for eight years after the initial surgical resection and has had no recurrence in the two years after the most recent surgery (the patient turned 70 years old).

\section{Discussion}

Liposarcomas commonly occur in the extremities, particularly in the thigh; however, primary pleural liposarcoma is very rare with very few reports of isolated cases. ${ }^{1-4)}$ Although the exact incidence of primary pleural liposarcoma is unknown, an intrathoracic origin liposarcoma comprises $2.7 \%$ of all liposarcoma cases and most liposarcomas in this region arise in the mediastinum. ${ }^{5)}$ In fact, Matsukuma et al. conducted a literature review of primary pleural liposarcomas in 2019, reporting only 31 cases of primary pleural liposarcoma published from 1942 to 2018. ${ }^{3)}$ Furthermore, a literature search in PubMed using keywords such as "pleura" and "liposarcoma" from 2018 to 2021 yielded only four other case reports of primary pleural liposarcoma.

The World Health Organization has classified liposarcoma into five types: atypical lipomatous tumor, well-differentiated liposarcoma, dedifferentiated liposarcoma, myxoid liposarcoma, and pleomorphic liposarcoma. ${ }^{6)}$ Of these, dedifferentiated liposarcoma in particular has a significantly poor prognosis with considerable rates such as $41 \%$ of cases developing local recurrence; $17 \%$, metastasis; and only 28\%, 5-year survival. In contrast, the 5-year survival rate of welldifferentiated liposarcoma is $80 \%$. $^{7)}$ The curative treatment for liposarcoma is prompt surgical resection with chemotherapy; radiation therapy and chemoradiotherapy are occasionally combined as adjuvant therapy. ${ }^{4)}$ However, adjuvant chemotherapy is selectively used because the subtypes differ in chemosensitivity. In cases of the resistant subtype of dedifferentiated liposarcoma, chemotherapy is not indicated. ${ }^{8)}$ Radiation therapy can reportedly reduce the risk of local recurrence, especially in myxoid liposarcoma. ${ }^{9)}$

In terms of surgical resection of tumors at the cavoatrial junction, Neves et al. classified the venous tumor thrombus into four categories: level I - renal vein thrombus, level II - infrahepatic IVC thrombus, level III retrohepatic IVC thrombus, and level IV - intraatrial IVC thrombus. ${ }^{10)}$ In this case, the cavoatrial tumor thrombus can be classified as level IV. The standard surgical technique for levels III and IV tumor thrombi is 
Matsumoto $R$, et al.

resection with $\mathrm{CPB}$. However, the use of $\mathrm{CPB}$ for patients with malignant diseases is controversial because $\mathrm{CPB}$ decreases patients' immunity and potentially spreads cancer cells systemically. ${ }^{11)}$ Furthermore, CPB is associated with the release of inflammatory mediators, coagulopathy, platelet dysfunction, and increased bleeding. Reports of the use of CPB in cancer patients have demonstrated varying levels of support. ${ }^{12,13)}$ Suzuki et al. reported that among 74 patients with metastatic cancer undergoing open heart surgery with and without CPB, no significant difference was observed in cancer-specific mortality (patients with and without CPB: $26.7 \%$ and $24.1 \%$, respectively; $\mathrm{p}=0.8) .{ }^{12)}$ Conversely, Pinto et al. reported that among 43347 patients undergoing isolated CABG surgery, the adjusted relative risk of cancerspecific mortality caused by CPB was 1.16 (95\% confidence interval: $0.92-1.46 ; \mathrm{p}=0.20){ }^{13)}$ Consequently, although some association between $\mathrm{CPB}$ and cancer progression might exist, we did not consider it significant enough to warrant aggressive surgical resection with CPB.

Similarly, concerning the use of HCA, there have been no reports of its use in cavoatrial tumors; in particular, no liposarcoma was reported. Zhu et al. reported that CPB with HCA to treat level III or IV renal or adrenal tumors enabling complete tumor resection did not increase the operative risks and was relatively safe. ${ }^{14)}$ The HCA facilitated complete cavoatrial tumor removal and the inside of the IVC could be assessed endoscopically.

Complete resection of a cavoatrial metastatic tumor with thrombectomy, which became feasible through using $\mathrm{CPB}$ with $\mathrm{HCA}$, might contribute to mid-term survival without the recurrence of poor-prognostic liposarcoma.

\section{Conclusion}

Prompt complete resection of the cavoatrial metastatic liposarcoma associated with poor prognosis was successfully performed using CPB with HCA, with subsequent satisfactory mid-term survival without recurrence.

\section{Informed Consent}

The patient provided informed consent.

\section{Disclosure Statement}

All authors declare that they have no conflicts of interest.

\section{References}

1) Crago AM, Brennan MF. Principles in management of soft tissue sarcoma. Adv Surg 2015; 49: 107-22.

2) Wong WW, Pluth JR, Grado GL, et al. Liposarcoma of the pleura. Mayo Clin Proc 1994; 69: 882-5.

3) Matsukuma S, Oshika Y, Utsumi Y, et al. Pleural dedifferentiated liposarcoma: a case report. Mol Clin Oncol 2019; 10: 132-6.

4) Rizer M, Singer AD, Edgar M, et al. The histological variants of liposarcoma: predictive MRI findings with prognostic implications, management, follow-up, and differential diagnosis. Skeletal Radiol 2016; 45: 1193-204.

5) Kara M, Ozkan M, Dizbay Sak S, et al. Successful removal of a giant recurrent mediastinal liposarcoma involving both hemithoraces. Eur J Cardiothorac Surg 2001; 20: 647-9.

6) Fletcher CD, Bridge JA, Hogendoorn PC, Mertens F, eds. WHO Classification of Tumours of Soft Tissue and Bone, 4th ed. Lyon: IARC Press, 2013; 342.

7) Henricks WH, Chu YC, Goldblum JR, et al. Dedifferentiated liposarcoma: a clinicopathological analysis of 155 cases with a proposal for an expanded definition of dedifferentiation. Am J Surg Pathol 1997; 21: 271-81.

8) Crago AM, Singer S. Clinical and molecular approaches to well differentiated and dedifferentiated liposarcoma. Curr Opin Oncol 2011; 23: 373-8.

9) Crago AM, Dickson MA. Liposarcoma: multimodality management and future targeted therapies. Surg Oncol Clin N Am. 2016; 25: 761-73.

10) Neves RJ, Zincke H. Surgical treatment of renal cancer with vena cava extension. Br J Urol 1987; 59: $390-5$.

11) Évora PRB, Albuquerque AAS, Nadai TR, et al. The cardiopulmonary bypass and cancer dissemination puzzle1. Acta Cir Bras 2018; 33: 1037-42.

12) Suzuki S, Usui A, Yoshida K, et al. Effect of cardiopulmonary bypass on cancer prognosis. Asian Cardiovasc Thorac Ann 2010; 18: 536-40.

13) Pinto CA, Marcella S, August DA, et al. Cardiopulmonary bypass has a modest association with cancer progression: a retrospective cohort study. BMC Cancer 2013; 13: 519.

14) Zhu P, Du S, Chen S, et al. The role of deep hypothermic circulatory arrest in surgery for renal or adrenal tumor with vena cava thrombus: a single-institution experience. J Cardiothorac Surg 2018; 13: 85. 